\title{
An Improved Mnemonic Device for Thermodynamic Relations
}

\author{
Younghun $\mathrm{Kim}^{1}$ and Rengaraj Selvaraj ${ }^{2 *}$
}

\author{
${ }^{1}$ Department of Chemical Engineering, Kwangwoon University, Seoul 139-701, Korea. \\ ${ }^{2}$ Department of Chemistry, College of Science, Sultan Qaboos University, P.O. Box: 33, \\ Al Khoud, PC 123, Muscat, Sultanate of Oman. *E-mail: Rengaraj@squ.edu.om.
}

\begin{abstract}
An improved mnemonic device for thermodynamic relations between state variables and potentials was proposed in the form of a thermodynamic circle. Based on separating the Born square into an inner square ( $\mathrm{T}$, $\mathrm{P}, \mathrm{V}$, and $\mathrm{S})$ and an outer circle $(\mathrm{G}, \mathrm{A}, \mathrm{U}$, and $\mathrm{H})$, relations such as Legendre transforms, Maxwell equations, equations to compute variables, and differential equations for thermodynamic variables can be recalled easily. The thermodynamic circle has a cross-arrow at its center and can be used to intuitively determine the sign of all thermodynamic relations.
\end{abstract}

Keywords: Chemical Engineering; Mnemonics; Thermodynamics; Maxwell equations.

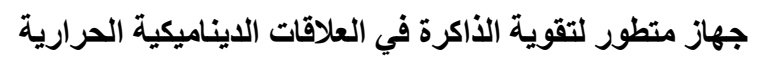

يونجهن كيم و رينجراج سلقراج

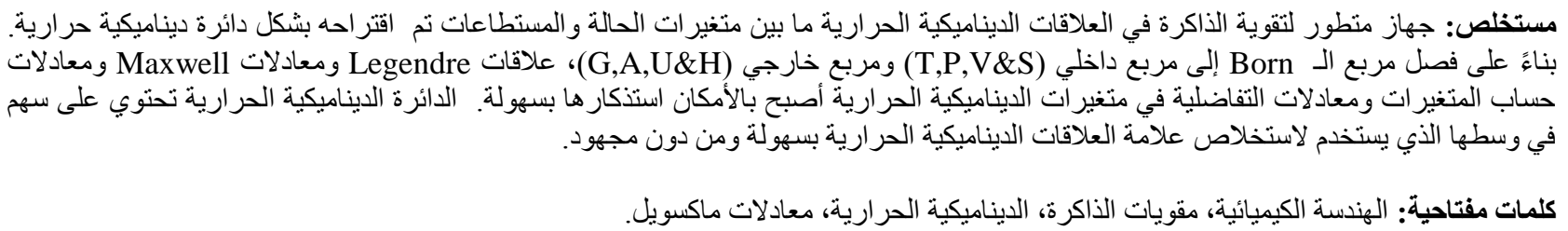

\section{Introduction}

$\mathbf{V}$

arious relations, such as Legendre transforms, Maxwell equations, equations to compute variables, and differential equations for variables, are found in thermodynamics [1-3]. Despite their best efforts in studying the actual logic that underlies the thermodynamic relations, many students have trouble remembering these relations between the four state variables (temperature, T; pressure, P; volume, V; entropy, S) and the four thermodynamic potentials (internal energy, U; Helmholtz energy, A; enthalpy, H; Gibbs energy, G). Therefore, several mnemonic schemes to help recall the thermodynamic relations based on the famous Max Born square [1-5] have been developed.

Using the Max Born square, a three-dimensional extension was proposed by Phillips [3] and a similar thermodynamic cube was suggested by Pate [2]. A simpler mnemonic diagram for thermodynamic relationships was also proposed by Rodriguez and Bralnard [5] and was modified as several mnemonic patterns [4]. As an improved mnemonic tool, a circle type named 'thermodynamic circles' was also proposed by Natarajan [1]. Other diagrams such as an energy pie have been suggested to provide differential expressions of the potentials as well as Maxwell relations $[6]$.

However, these schemes still require complicated rules to remember the positive or negative signs in front of the terms in the thermodynamic relations [4,5]. Three-dimensional cube [2] or octahedral mnemonic devices [3] are complicated and also difficult to remember. To overcome this drawback, separating the Max Born square into two sections was suggested, and this makes recalling the equations much more straightforward [7]. The Zhao model is a very powerful tool compared to the others, but decision as to sign (+ or - ) still remains challenging.

Therefore, the mnemonic circle found in the thermodynamic field is suggested here based on separating the Max Born square into an inner square (T, P, V, and S) and an outer circle ( $\mathrm{G}, \mathrm{A}, \mathrm{U}$, and $\mathrm{H})$. The thermodynamic circle has a cross-arrow at its center and this constitutes a key component for determining the sign of each of the relations. This cross-arrow constitutes an improvement upon the mnemonic device proposed by Zhao [7] and enables one to determine the signs of thermodynamic variables intuitively . 


\section{Suggested mnemonic}

As shown in Figure 1(a), the thermodynamic circle is composed of three components; an inner square (four state variables), an outer circle (four thermodynamic potentials), and a cross-arrow (sign determination). The direction of the cross-arrow determines the signs of all relations, and thus it acts as a vector which is fully described by both a magnitude and a direction. For example, when the path of some relation follows the reverse direction to the TS arrow, it has a negative sign in the target relation. The product of TS and PV has same dimension unit as thermodynamic potentials with outer circle $(\mathrm{G}, \mathrm{A}, \mathrm{H}$, and $\mathrm{U})$.
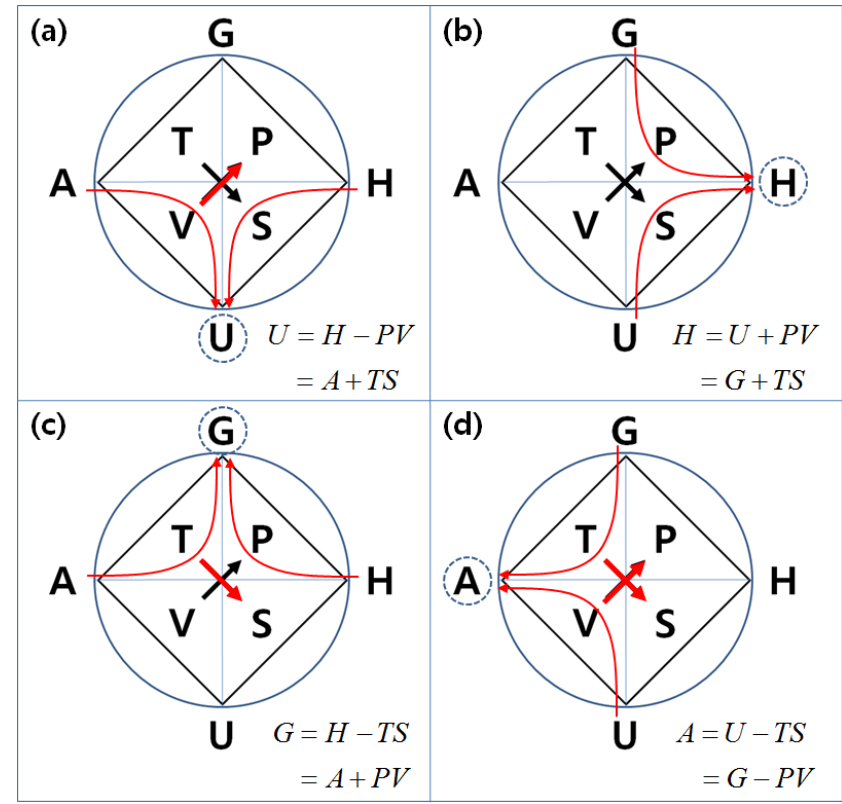

Figure 1. Scheme to recall the Legendre transforms for four thermodynamic potentials.

\section{Use of the mnemonic tool}

\subsection{Relations between neighboring potentials ( $\mathrm{U}, \mathrm{A}, \mathrm{H}$, and $\mathrm{G})$}

Legendre transforms of the four thermodynamic potentials are determined in the following way. As shown in Figure 1a, internal energy $(U)$ is separately formed by two different neighboring potentials,. By drawing a curved arrow from one corner of the potential to target potential, passing by the nearer center-arrow (TS or VP) diagonally, the target potential has two relations. For example, the curved arrow from $\mathrm{H}$, passing by the nearby VP arrow (opposite direction, therefore negative sign), forms $\mathrm{U}$ equal to $\mathrm{H}-\mathrm{PV}$. In addition, the curved arrow from A, passing by the nearby TS arrow (same direction, therefore positive sign) forms $\mathrm{U}$ equal to A + TS. Other potentials (H, G, and A) are formulated in the same way, as in with Figures 1b-1d. With intuitive decision of the sign from the directions of TS and VP, Legendre transforms of the four potentials can be recalled easily as $\mathrm{U}=\mathrm{H}-\mathrm{PV}=\mathrm{A}+\mathrm{TS}, \mathrm{H}=\mathrm{U}+\mathrm{PV}=\mathrm{G}+\mathrm{TS}, \mathrm{G}=$ $\mathrm{H}-\mathrm{TS}=\mathrm{A}+\mathrm{PV}$, and $\mathrm{A}=\mathrm{U}-\mathrm{TS}=\mathrm{G}-\mathrm{PV}$.

\subsection{Differential forms of the potentials}

The differential expressions ( $\mathrm{dU}, \mathrm{dA}, \mathrm{dH}$, and $\mathrm{dG}$ ) for the four thermodynamic potentials are easily determined by drawing two curved arrows. As shown in Figure 2a, two curved arrows depart from $\mathrm{P}$ and $\mathrm{T}$ toward the derivative of the target potential (dU) crossing at the intersection of the two arrows (nearby center-arrow), following which the direction of the curves TdS and PdV mirror each other. Thus, $(+\mathrm{TdS}-\mathrm{PdV})$ gives dU. In the same fashion, Figures $2 \mathrm{~b}$ $-2 \mathrm{~d}$ provide $\mathrm{dH}=\mathrm{TdS}+\mathrm{VdP}, \mathrm{dG}=-\mathrm{SdT}+\mathrm{VdP}$, and $\mathrm{dA}=-\mathrm{SdT}-\mathrm{PdV}$. Again, all signs $(+$ or - ) of derivatives are determined by the direction of the TS and VP arrows. 

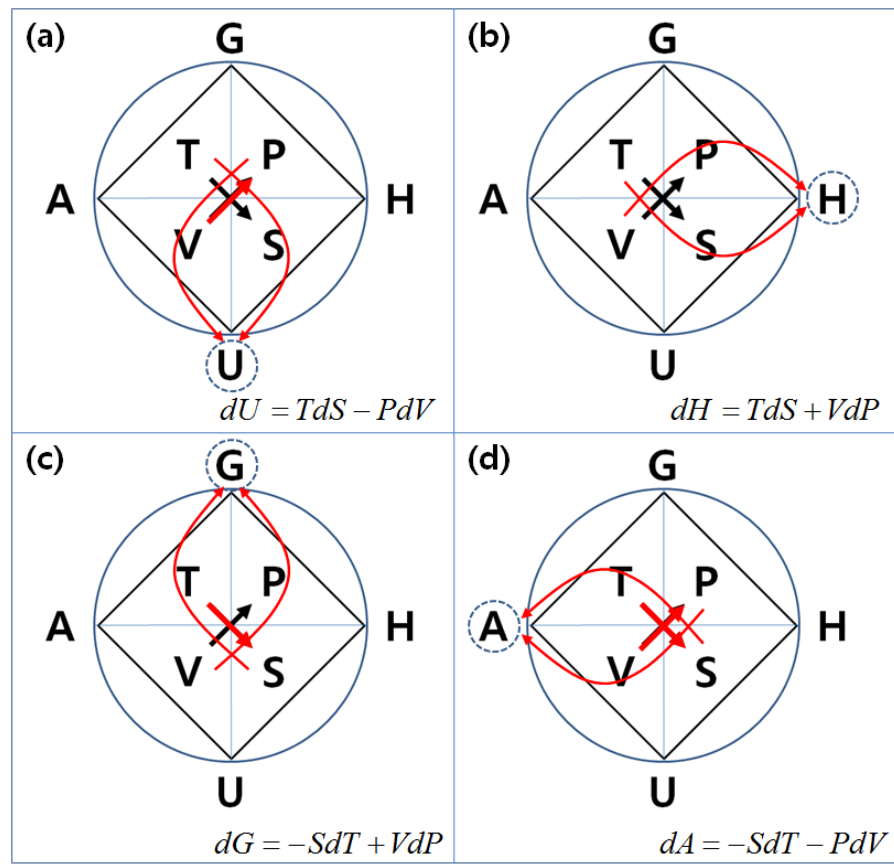

Figure 2. Scheme to recall the differential form of four thermodynamic potentials.

\subsection{Relations for compute variables (T, P, V, and S)}

One can also remember the relations for thermodynamic state variables from the partial derivatives of the thermodynamic potentials. This is schematically shown in Figure 3, and the sign of the partial differential of a potential with respect to the conjugate variable is again determined by the TS and VP arrows. For example, as shown in Figure 3a, two curved arrows depart from $\mathrm{T}$ and pass through $\mathrm{S}$, then reaching the nearby potentials $(\mathrm{H}$ and $\mathrm{U})$ and finally terminating in the reverse direction $(\mathrm{P}$ and $\mathrm{V})$. Thus the two curved arrows give $\mathrm{T}=(\partial \mathrm{H} / \partial \mathrm{S})_{\mathrm{P}}=(\partial \mathrm{U} / \partial \mathrm{S})_{\mathrm{V}}$. Since the two curved arrows follow the same direction as the TS arrow, the partial differential has a positive sign. Similarly, other variables can be recalled, based on Figures $3 \mathrm{~b}-3 \mathrm{~d}$, as $\mathrm{V}=(\partial \mathrm{H} / \partial \mathrm{P})_{\mathrm{S}}=(\partial \mathrm{G} / \partial \mathrm{P})_{\mathrm{T}}, \mathrm{S}=-(\partial \mathrm{G} / \partial \mathrm{T})_{\mathrm{P}}=-(\partial \mathrm{A} / \partial \mathrm{T})_{\mathrm{V}}$ and $\mathrm{P}$ $=-(\partial \mathrm{A} / \partial \mathrm{V})_{\mathrm{T}}=-(\partial \mathrm{U} / \partial \mathrm{V})_{\mathrm{S}}$.
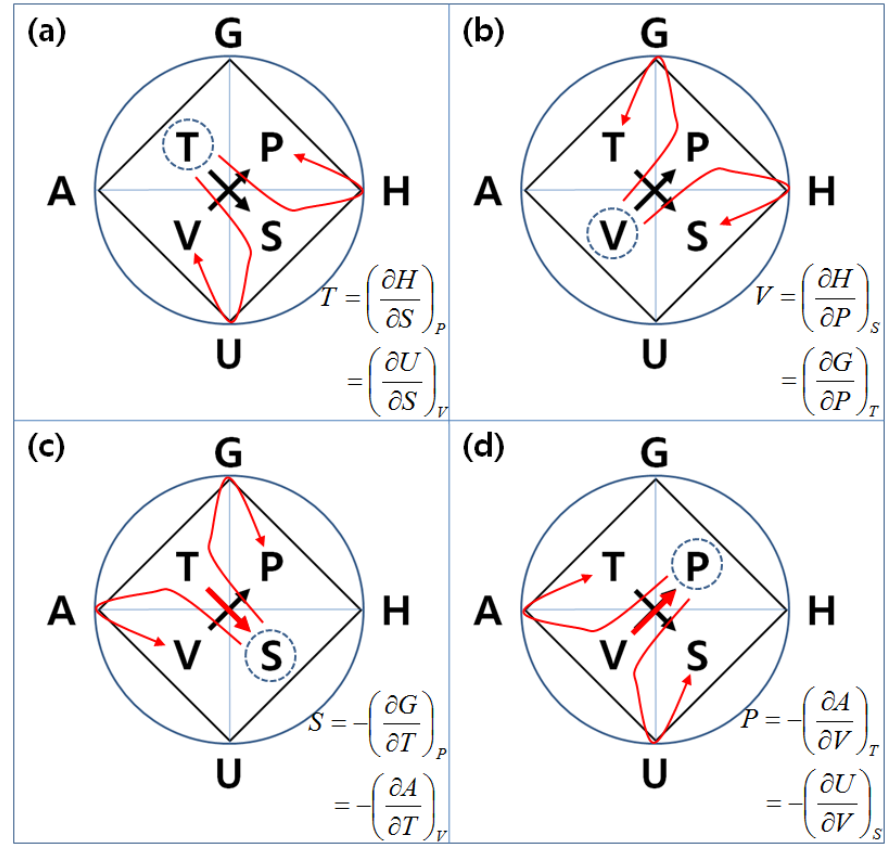

Figure 3. Scheme to recall the compute variables from partial differentials of four thermodynamic potentials with respect to the conjugate variables. 


\subsection{Maxwell relations}

The Maxwell relations have the form of partial derivatives of the four thermodynamic state variables $(\mathrm{T}, \mathrm{P}, \mathrm{V}$, and S), and, with the same diagrams, one can also easily remember the Maxwell equations using simple schemes (Figure 4). In a similar way to that above, two curved arrows separately depart from $\mathrm{T}$ and $\mathrm{P}$, pass through $\mathrm{V}$ and $\mathrm{S}$ to reach S and V respectively (Figure 4a). Since the direction of the PSV curve is opposite to the VP arrow, the partial derivative has a negative sign. Namely, this yields the partial derivative of $\mathrm{T}$ (or P) with respect to $\mathrm{V}$ (or $\mathrm{S}$ ) at constant $\mathrm{S}($ or $\mathrm{V})$, and so one can obtain $(\partial \mathrm{T} / \partial \mathrm{V})_{\mathrm{S}}=-(\partial \mathrm{P} / \partial \mathrm{S})_{\mathrm{V}}$. Other Maxwell equations can be obtained as shown in Figures $4 \mathrm{~b}-4 \mathrm{~d}:(\partial \mathrm{V} / \partial \mathrm{S})_{\mathrm{P}}=(\partial \mathrm{T} / \partial \mathrm{P})_{\mathrm{S}} ;(\partial \mathrm{V} / \partial \mathrm{T})_{\mathrm{P}}=-(\partial \mathrm{S} / \partial \mathrm{P})_{\mathrm{T}} ;-(\partial \mathrm{P} / \partial \mathrm{T})_{\mathrm{V}}=-(\partial \mathrm{S} / \partial \mathrm{V})_{\mathrm{T}}$
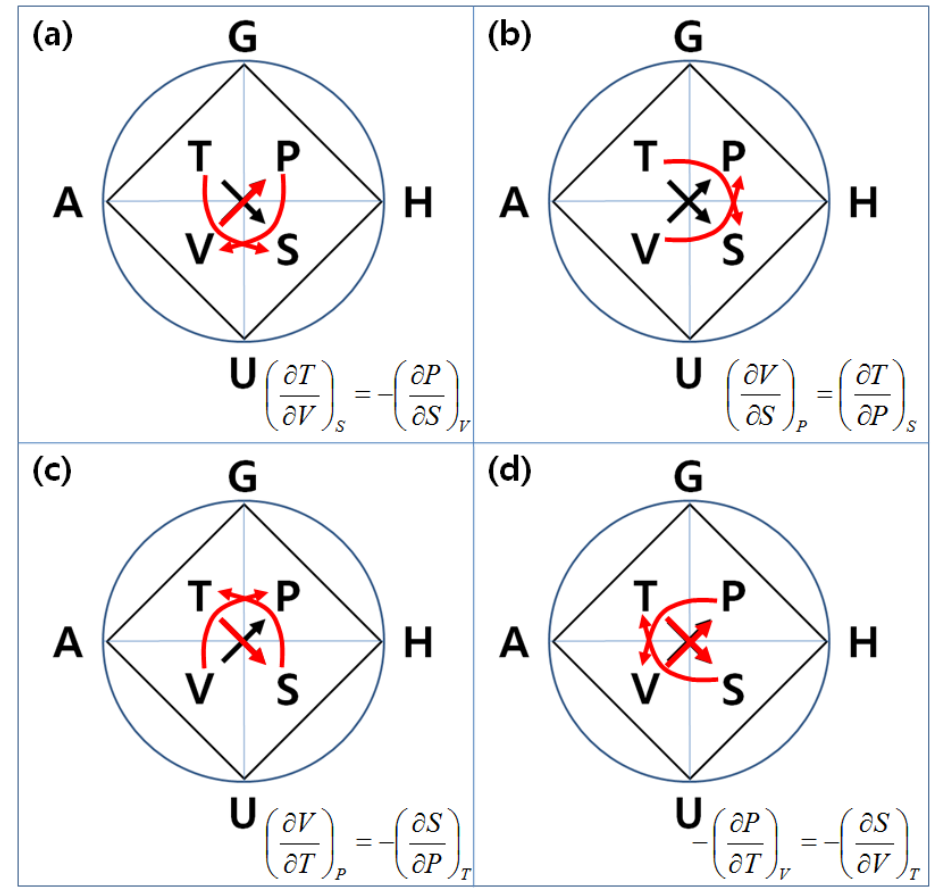

Figure 4. Scheme to recall the Maxwell relations involving the four thermodynamic state variables.

\section{Impact on student learning}

To confirm the real effectiveness and efficiency of this tool for the recall of thermodynamic relations, a serial test was carried out on junior students (98) in college. Firstly, after teaching the actual logic of thermodynamic relations (Legendre transforms, Maxwell equations, equations to compute variables, and differential equations for thermodynamic variables), a test of their memorization of the 4 relations was conducted. Most of the students only recalled Legendre transforms. As the next step, the Zhao model was used to recall the thermodynamic relations. About $29.6 \%$ of students succeeded in recalling the 4 relations within $10 \mathrm{mins}$, but the signs in front of the terms were still confused. Finally, the mnemonic tool proposed herein was taught to students, and ca. $77.5 \%$ of the students were found to have memorized all the relations perfectly. The students were surprised at themselves for recalling the thermodynamic relations so accurately and readily.

\section{Conclusion}

The thermodynamic circle is a mnemonic device that allows for easier recall of the thermodynamic relations between thermodynamic state variables and potentials. Separating the Max Born square into two components was first suggested by Zhao, and the circle-type mnemonic device suggested here is an improved form. The thermodynamic circle has a cross-arrow at its center which is helpful to intuitively determine the sign of various terms (sign of TS/VP, partial derivatives, and Maxwell equations). The author would like to add a whimsical tip for recalling the energy conservation law. Most of us use the qwerty keyboard daily, and we can find the following conservation law equation at our fingertips: $\mathrm{Q}+\mathrm{W}=\mathrm{E}=\mathrm{RT}$. 


\section{RENGARAJ SELVARAJ and YOUNGHUN KIM}

\section{References}

1. Natarajan, K., Graphical summaries for Maxwell relations of closed and open thermodynamic systems. Inter. J. Mech. Eng. Edu., 2014, 42, 1-17.

2. Pate, S.F., The thermodynamic cube: a mnemonic and learning device for students of classical thermodynamics. Am. J. Phys., 1999, 67, 1111-1113.

3. Phillips, J.M., Mnemonic diagrams for thermodynamic systems. J. Chem. Edu., 1987, 64, 674-675.

4. Pogliani, L. and La Mesa, C., The mnemonic diagram for thermodynamic relationships. J. Chem. Edu., 1992, 69, 808-809.

5. Rodriguez, J. and Bralnard, A.J., An improved mnemonic diagram for thermodynamic relationships. J. Chem. Edu., 1989, 66, 495-496.

6. Fieberg, J.E. and Girard, C.A., Mnemonic device for relating the eight thermodynamic state variables: the energy pie. J. Chem. Edu., 2011, 88,1544-1546.

7. Zhao, J.C., A mnemonic system for thermodynamics. MRS Bull., 2009, 34, 92-94.

Received $1^{\text {st }}$ August 2016

Accepted 4 September 2016 